\title{
Rebuilding trust: novel standards for reporting the effectiveness of male-pattern hair loss treatment
}

\begin{abstract}
As photo editing software makes rapid progress, researchers and clinicians have faced the almost irresistible seduction of manipulating their work result in the form of pictures. As a result, academic journals have made huge efforts to ensure the authenticity of the photos included in manuscripts, going so far as to develop software for fabrication detection. Yet the clinical field of healthcare has not had the bandwidth to carry out such inspections, probably because the need for self-purification was not as great as in academia and treatment effectiveness more often than not is demonstrated through pre- and post-treatment photo comparisons, which significantly influences the reputation of the organization or individual care provider. It is time to change. The healthcare industry should provide the most precise and truthful information that patients have long deserved. We believe the most effective and efficient way to encourage authentic treatment effectiveness reporting is to develop a well-designed guideline that every caregiver can follow. To make a solid example, and as hair loss treatment specialists, we develop the Total View Guidelines to clearly show the effectiveness of androgenic alopecia treatment. To ensure maximum authenticity, instead of using an easily manipulative photo as the modality, we adopt video recording. Through an approximately two-minute recording, the Total View Guidelines compels no interruption-in other words, it prohibits pushing the pause button. This uninterrupted video and the maneuvers showing the patient's hair and scalp make the Total View Guidelines a reliable, objective, and non-manipulative guideline. We trust our colleague care givers; only a handful of them manipulate treatment results, and such misbehavior has made the entire healthcare appear untrustworthy. We hope that everybody can use this guideline in order to open the gate widely, welcoming the trust that the healthcare industry might have temporarily lost.
\end{abstract}

Volume 4 Issue I - 2016

\author{
Hyun Wook Baik,' Heon-Jae Jeong² \\ 'The NobleLine Center for Alopecia Care, Seoul, Korea \\ ${ }^{2}$ The Care Quality Research Group, Baltimore, USA
}

Correspondence: Heon-Jae Jeong, The Care Quality Research Group, 624 North Broadway, Rm. 455, Baltimore, MD, 21205 , USA, Tel + I-4l 0-733-2452, Fax + |-4l 0-955-6959, Email hj957I@gmail.com

Received: July 8, 2016 | Published: July II, 2016

\section{Introduction: the devil wears photoshop}

In 2005, the scientific world was immensely shocked and traumatized by the notorious scandal of Dr. Woo Suk Whang's photo manipulation including in his famous articles about embryonic stem cell research published in Science, one of the most prestigious academic journals. Indeed, his team made an even bolder move than simply re-touching photos depicting their study results; they actually did a copy-and-paste adjustment to some pictures. Luckily for academia as a whole, the manipulation was caught before the studies led countless future researchers in the wrong direction. Yet it was not the journal, Science, that called out the fraud, but an Internet community called Biological Research Information Center (BRIC), voluntarily run by Korean scholars and students. Science never even questioned the authenticity of the photos in Dr. Whang's manuscript.

At the time of Dr. Whang's publication - only about a decade ago-academic journals did not feel the need to investigate potential fraud, and scientists did not hesitate to trust each other's study results: It was a golden age, when researchers believed in concepts like honesty, ethics, and camaraderie in academia. Today, this situation has completely changed. Ignited by Dr. Whang's and others' unforgivable cases, academia began to take actions. For example, The Journal of Cell Biology scrutinizes all articles accepted for the journal and has reported that approximately 25 percent of them contained at least one manipulated illustration. ${ }^{1,2}$ Such a finding shocked the scientific arena even more, and scientists asked one another what was happening, why, and how.

At the center of this turmoil is photo editing software such as Photoshop, which enables researchers to easily alter photos to favor their agenda in academic articles, as evidenced in Dr. Whang's case. Photoshop, one of the most convenient tools in our daily lives that were supposed to make our family look prettier, ultimately sounded the death knell for the once fraud-proof modality for reporting research results. After the funeral for non-manipulable photos, some of the bereaved changed sides, selling their souls to the devil. At first, they just made small changes to photos. But soon one thing led to another, and individuals ended up manipulating all aspects of photos, to the point that we could no longer believe our eyes, like with the digitally added dinosaurs in the movie Jurassic Park.

Declaring a holy war on such manipulation, academic journals began to develop guidelines - or, rather, requirements - for photo submissions to prevent such fraud. ${ }^{3}$ They even tried to develop computer applications for detecting almost indistinguishable photo manipulation. Regardless of whether these efforts were successful or not, it is truly unfortunate that many resources that could have been invested to lead us to scientific revolutions are being wasted to patrol for unethical acts of fabrication guided by Mephistopheles in scientific research.

Photos are critical not only for laboratory-level research, but also the clinical field, where healthcare services are provided for patients. Patients always want to see examples of the treatment effectiveness they can expect with their own eyes before making a decision about the type of treatment and who will provide it. Photos are undoubtedly the major vehicle for showing treatment results to patients who might not be able to understand complicated medical terminology.

To everyone's sorrow, some caregivers have left us for the land where the head of the devils dominate: Not surprisingly, there is much more temptation to engage in photo manipulation in clinical settings, 
since being chosen by patients is the most important determinant of a healthcare organization's or an individual care provider's financial security. In this wild land, even caregivers at the highest ethical levels are easily trapped in an Oscar Wilde-inspired state of mind: "I can resist anything except temptation."

Something had to be done.

Driven by moral obligation and helped by numerous colleagues, we developed an idea in the form of guidelines to avert such photo manipulation in treatment effectiveness reporting. We introduce these guidelines here. Although we use one clinical condition, androgenic alopecia (i.e., male-pattern hair loss), as an example, the essence and approach shown in this article can easily be applied to various treatment methods over many clinical conditions.

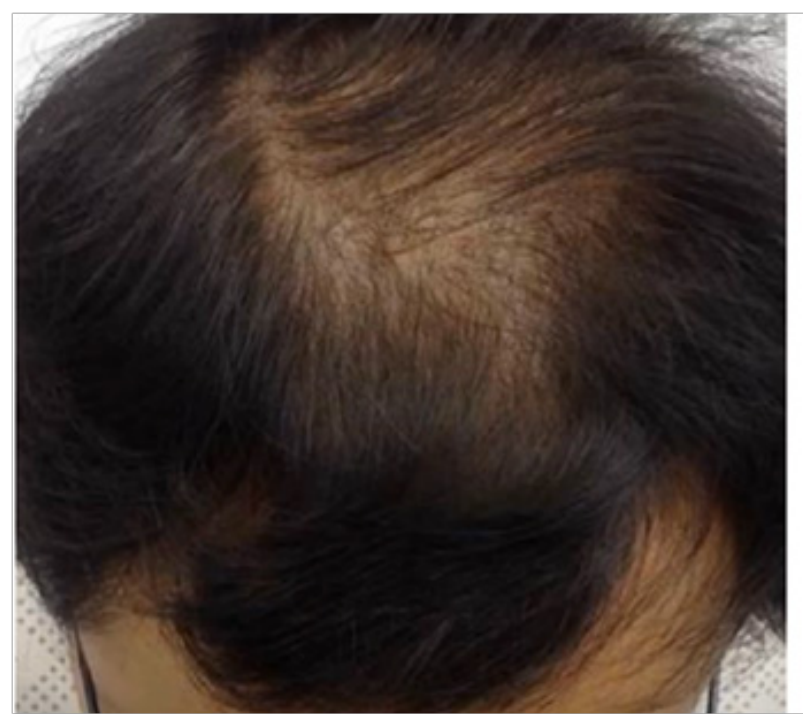

\section{Alopecia: where lucifer would love to reside}

Male-pattern hair loss is a highly prevalent disease, ${ }^{5}$ and numerous treatment options are available, ranging from sophisticated hair transplant surgery to aesthetic products like hair loss concealing powder. However, no matter what treatment method is chosen, few alternatives exist for reporting treatment effectiveness other than photos. Such a monopolistic status of photos has made alopecia care one of the clinical realms where the most irresistible temptation of photo manipulation is seducing care providers. To share some hints on what kind of fabrication is being done in the real world, we show a typical before and after comparison pictures of alopecia treatment (Figure 1).

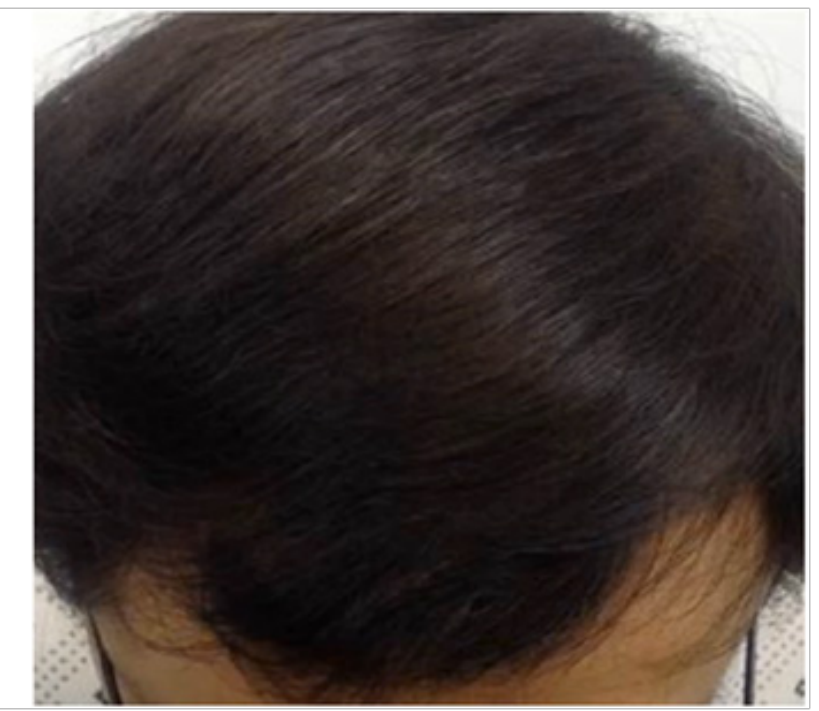

Figure I Fake before and after treatment photos made only by different combing directions.

At first sight, assuming the one of the left is the before photo and the one on the right is the after photo, these pictures apparently indicate the greatest possible success in alopecia treatment. However, there is a problem: These pictures were actually taken on the same day on the same patient. The only difference was the combing direction used. Indeed, there are many other ways to commit forgery without using any special instruments, such as using different camera angles or lighting while taking pre- and post-treatment photos, taking the post-treatment photo with more hair, wetting the hair in the pretreatment shot to make it look like there is less hair, and parting the hair in different locations to exaggerate hair loss in the pre-treatment picture. As Figure 1 proves, these simple manipulative maneuvers can dramatically change our perceptions of the effectiveness of a treatment. Naturally, if sophisticated methods like photo-editing software are fully mobilized, even an image expert may not be able to discern the authenticity of the photos. The fact that such manipulation is astonishingly simple and easy might make the alopecia treatment industry Lucifer's most beloved land, where his quiet whisper can readily turn anybody into a demon's associate.

At this point, we seem to have every right to be suspicious and to make biting remarks to all caregivers treating hair loss. The former reaction might be reasonable, but the latter is completely unfair: The majority of hair loss caregivers are honest and righteous, always putting their patients first and brushing off Lucifer's whisper. We have no right to wrongfully accuse these beautiful minds.

Then, what do we need to do? The first step is to develop a standard for reporting alopecia treatment results, as the previously mentioned scientific journals did. Once we promulgate such a standard and convince every caregiver to use it, comparing results between caregivers and between treatment methods will be much more objective - not to mention, the alopecia industry will be able to provide patients with trustworthy before and after treatment comparisons. The question is whether we can develop such a standard that is objective, reliable, easy to apply in everyday life, and (most importantly) non-manipulative. Our answer is a resounding yes - in fact, it is "yes, we did."

\section{The total view project: the ultimate in reporting male-pattern hair loss treatment results}

There is a simple rule: The more information a medium has, the more difficult it is to manipulate it. For example, text reports have historically been very easy to fabricate whereas photos were more difficult to manipulate. As recently as a couple of decades ago photos were said to be non-manipulable. Yet, as we have seen, 
photos are no longer a fabrication-clean medium; therefore, we must take a leap to the next generation information container. We chose motion pictures - namely, video. As of today, video is not easily manipulated, and it can show the whole scalp and hair thoroughly, including changes in camera angles and combing processes in real time without any discontinuity. Therefore, video reporting can prevent all the photo fabrication tricks introduced thus far, showing the most realistic description of treatment results. We developed a standardized procedure for reporting video results, which we named the Total View Guidelines. As part of the Total View Project, several hospitals have been participating in this noble movement to clear their name by corroborating in an unprecedented level of authenticity in treatment results.

Before we introduce the Total View Guidelines (version 1.0), we would like to make it clear that the Total View approach does not require any special equipment or additional time. The following guidelines detail the required or recommended instruments for conducting Total View; most of them are already readily available to any caregiver.

You might think that the equipment list is too simple for a treatment reporting method claiming maximum reality and authenticity. It is a legitimate concern that we had as well. We actually experimented with many kinds of professional filming equipment available on the market. After comparing the results from countless combinations, we were convinced that the list provided herein is more than sufficient for clearly reporting treatment results. As such, we kept pursuing such minimalism, which would ensure that caregivers could easily implement the Total View Guidelines without feeling an economic burden to purchase the equipment. Now, let us move on to the actual steps to follow when creating a Total View video.

\section{Instrument Requirements for Total View Guidelines Version I.0}

1. Tail comb: Any variants of tail comb work; 1-2 millimeter gaps between the comb's teeth are recommended (Figure 2). The tail is used for parting the hair.

2. Hair Band: In order to pull patient's hair back, a hair band with short comb teeth is required (Figure 2).

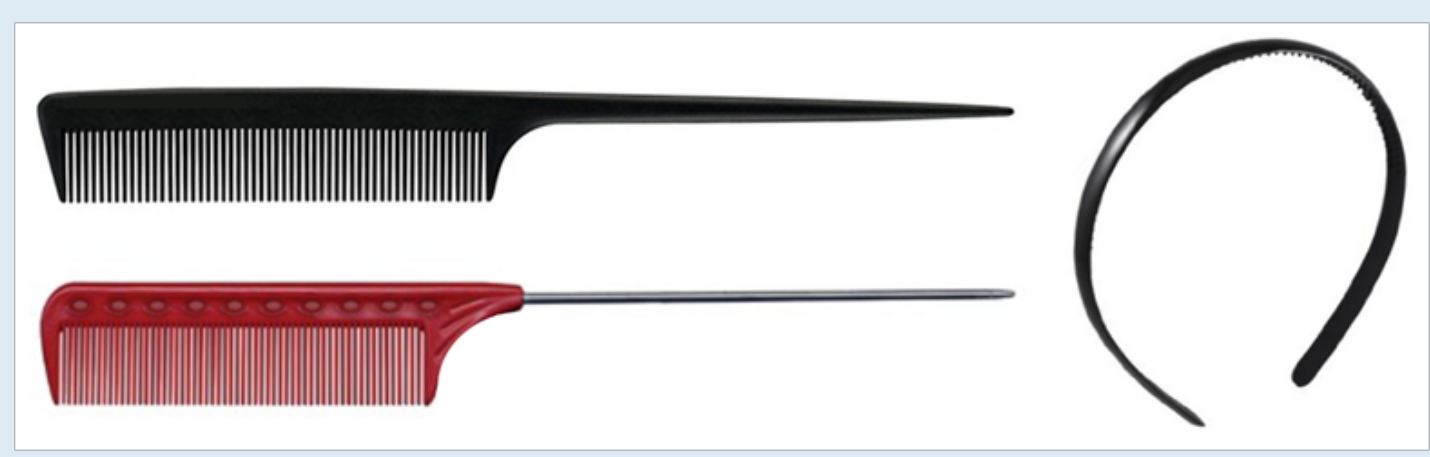

Figure 2 Examples of tail comb and hair band for the total view guidelines.

3. Swivel chair: Any swivel chair that can turn the patient 360 degrees will work. Avoid one that reclines because the patient should be kept in a stable position.

4. Video recorder: Any modern digital video recorder will work. A high definition (HD) recorder with a resolution of at least 720p is required; 1080p or higher is recommended. As of this writing, almost all quality smartphones have a built-in video recorder function with sufficient resolution.

5. Illumination: A rule of thumb is "brighter than an ordinary office." More technically, at least 500 lux is required; higher than 1,000 lux is recommended. In order to prevent shadows, at least two lamps from different directions are needed; if there is a third, the shadows on the wall can also be eliminated. For a hair transplant clinic, an operation room setting and its lighting meet this requirement. ${ }^{6}$

Thus far, the Total View Guidelines are the simplest but strongest way to show the effectiveness of male-pattern hair loss treatment in the most authentic way. Although the list of steps may appear a bit long, taking all the above steps takes no longer than 2 minutesand that investment is almost nothing compared to the amount of trust that the hair loss industry will regain. Before we move on, we should re-emphasize the importance of non-interrupted filming.
Whatever happens, filming all of the Total View steps should never be interrupted (paused) during the shooting because the non-stop filming plays a key role in proving the authenticity - manipulation free-of the video. Along the same lines, every maneuver, including combing and parting, should be conducted slowly and gently in order to clearly show the object, thereby guaranteeing full disclosure and proving that the caregiver did not attempt to hide anything. 


\section{Total View Guidelines (Version I.0)}

\section{General Information}

- The Total View comprises the evaluation of five different areas of a patient's scalp: hair line, frontal, mid-scalp, crown, and occipital and parietal areas (Figure 3).

- Once you begin to record, never stop or pause the recording. This is the essence of Total View that ensures the minimization of any possibility of manipulation.

- In addition, recording the combing or parting process itself is essential; while combing or parting, we can best observe the treatment results. In the case of combing, combing in the opposite direction of hair flow best reveals hair density of the area. Note that it is important not to push hair down to the scalp while combing, which interferes with showing the hair density well. Combing and parting should be conducted slowly and gently.

- For best results, recording the video by two caregivers is recommended: one for handling the video recorder and the other for combing and parting.

- Areas that were not treated can be skipped; for example, patients who received treatment in the crown area does not need hairline, frontal, and mid-scalp evaluation. However, step 6, occipital and parietal area evaluation (step 6), must be conducted in all cases because it provides information on past treatment history, such as hair transplant.

- At each step, make sure the object is in focus on your video recorder.

- In total, 90-120 seconds are sufficient for filming the Total View.

- An actual Total View video following the steps on the next page can be found at http://www.total-view.org/

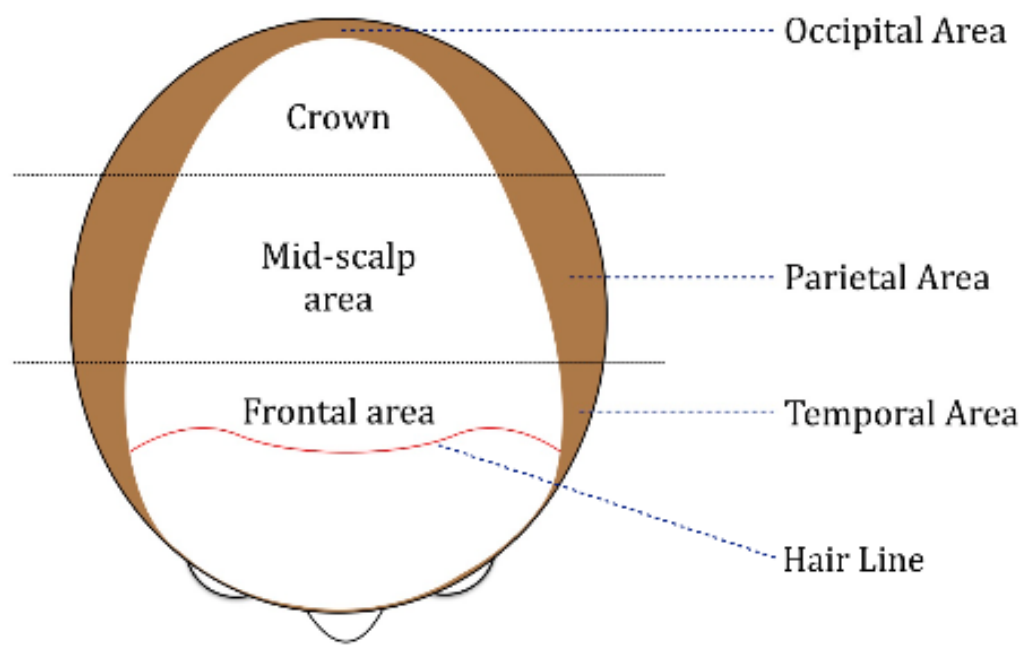

Figure 3 scalp area seen from above. 


\begin{tabular}{|c|c|c|}
\hline \multicolumn{3}{|r|}{ Step-by-step Description } \\
\hline 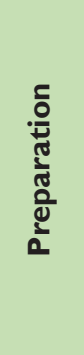 & 1) & $\begin{array}{l}\text { Preparation } \\
\text { - Wash the patient's hair thoroughly to remove any hair products, such as styling gel, spray, wax, and hair loss con- } \\
\text { cealing powder; these products obstruct smooth combing and clear parting. } \\
\text { - Seat patient on the swivel chair and set the video recorder to aim at the center of the patient's face. } \\
\text { - Adjust the exposure and white balance of the video recorder according to the filming environment, such as illumi- } \\
\text { nation. Video recorders with an automatic adjustment function often do not provide the results we expect; therefore, } \\
\text { checking these in person is a necessary step. } \\
\text { - Above all, maintain consistency in the video between pre- and post-treatment. This is critical. }\end{array}$ \\
\hline 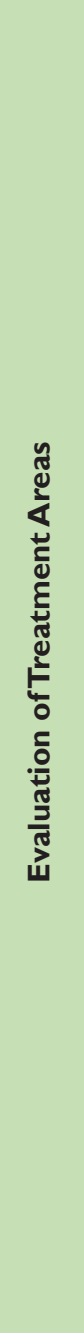 & 2) & $\begin{array}{l}\text { Evaluation of Hairline } \\
\text { - Put a hair band on the patient's head, pulling all hair back. } \\
\text { - Film hairline (frontal area) from three different angles (by turning the swivel chair): right } 45 \text { degrees, left } 45 \text { degrees, } \\
\text { and finally the exact center of the patient's head, lowered by } 45 \text { degrees. } \\
\text { - Put patient's head back into the original position and remove the hair band. } \\
\text { Evaluation of Frontal Area } \\
\text { - Lower the patient's head to the forward left direction to expose the right frontal area fully. } \\
\text { - Keep the distance between the hair and video recorder at around } 20-30 \text { centimeters ( } 8 \text {-12 inches); check the focus } \\
\text { of the video recorder and adjust as needed. } \\
\text { - Comb the right frontal area slowly } 3 \text { to } 4 \text { times. The combing direction is the opposite of the hair flow. } \\
\text { - Make a part in the right frontal area } 3 \text { to } 4 \text { times, showing the scalp in detail. } \\
\text { - Turn the patient's head to the center, while keeping the head down, and show the mid-frontal area using the combing } \\
\text { and parting techniques described above. } \\
\text { - Turn the patient's head to the right, while keeping head down, and show the left-frontal area using the combing and } \\
\text { parting techniques described above. } \\
\text { - Return the patient's head to the original position. } \\
\text { Evaluation of Mid-Scalp Area } \\
\text { - Lower the patient's head } 45 \text { degrees to show the mid-scalp area. } \\
\text { - Comb the mid-scalp area left and right. } \\
\text { - Comb in the opposite direction of hair flow. } \\
\text { - Make a part } 3 \text { to } 4 \text { times in the mid-scalp area, showing the scalp in detail. } \\
\text { - Return the patient's head to the original position. } \\
\text { - Comb the mid-scalp area left and right. } \\
\text { - Make a part } 3 \text { to } 4 \text { times in the crown area, showing the scalp in detail. } \\
\text { - Crown }\end{array}$ \\
\hline 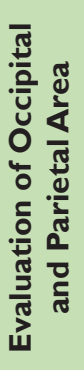 & 6) & $\begin{array}{l}\text { Evaluation of Occipital and Parietal Areas (Collecting Baseline Information) } \\
\text { - Turn the chair until the patient is facing backward (if step } 5 \text { was not taken), and lower the patient's head to look } \\
15 \text { degrees below the horizontal plane. } \\
\text { - Comb upward in different locations of the occipital area to show the scalp in detail. } \\
\text { - Turn the swivel chair counter-clockwise by } 15 \text { degrees and comb the left parietal area once. } \\
\text { - Do the same to the right parietal area. } \\
\text { - Return the patient's head to the original position and turn the chair until the patient faces forward once again. }\end{array}$ \\
\hline
\end{tabular}




\section{Be prepared: challenges the total view will face}

There can never be an everlasting or one-size-fits-all method to ensure the authenticity of treatment result reporting. Total View is no exception, and several challenges still have to be surmounted. First, any currently forgery-proof medium will eventually become something that can be manipulated, especially as computing power increases. It is only a matter of time. Although the Total View approach, with its uninterrupted video reporting, is a strong weapon now, it will eventually face challenges from video-manipulation technology, which will become as easy as photo editing. This means that the Total View Guidelines will need to be continuously updated according to technological evolution.

A more practical obstacle that the Total View must overcome is the difficulty in disseminating the guidelines. In many situations, medical treatments are the major remedy for a certain clinical condition; therefore, the dissemination of guidelines can be effectively achieved through hospitals or medical society. For alopecia, clinicians are the predominant caregivers using medically admitted therapeutics such as hair transplant surgery, low level laser therapy, and medications like Finasteride and Minoxidil. ${ }^{5,-9}$ However, many other professional positions provide remedies for hair loss, such as scalp masseur and masseuse and those who produce supplemental agents including depilatory cream, lotion, and hair loss concealing powder. Therefore, to disseminate the Total View Guidelines, we will have to access numerous channels to reach the various types of care providers.

Even if Total View Guidelines are successfully disseminated to all hair loss care providers, whether they actually implement them in their everyday work is a different story. Regardless of how good any guidelines are, care providers have shown a much lower propensity to embrace them than expected. Such a difficulty is embodied by the existence of the implementation science ${ }^{10}$ academic field. Thus, a variety of strategies have been tried to improve guideline implementation, ${ }^{11}$ some of which even include international collaboration. $^{12,13}$ To enhance the implementation of Total View Guidelines, it is worth mentioning the research of Jeong et al., ${ }^{14}$ which showed that the most important factors for guideline implementation is how easy they are to use and whether the workplace provides a supporting structure. ${ }^{14}$ Thus, developing a step-by-step checklist in the Total View Guidelines seems to be a promising way, as the effectiveness of checklists has been proved many times. ${ }^{15}$ Taking things one step further, we can develop an application (app) for smart phones and tablet PCs that provides real-time guides for the actions caregivers need to take (e.g., changing camera angle or patient's position) at specific moments while filming. In addition, this application can provide seamless integration from filming, storing, managing, and retrieving the videos.

As of this writing, we are developing a website for enhancing the dissemination and implementation of the Total View Guidelines. The site will also enable caregivers from around world to share their opinions on ways to improve the Total View Guidelines. A smart phone application has also been developed and is currently being tested.

\section{Conclusion: total view beyond alopecia}

This article has used only a single specific condition among the numerous diseases in various clinical fields as an example for consideration: male-pattern hair loss. To clarify, we had no intention of firing an aimed shot at the alopecia treatment field. Rather, we selected this example because we happen to be experts in hair loss treatment and quality of care specialists who believe that patients have every right to receive the most precise information about their treatment. Therefore, we should mention that our aspiration materialized by the Total View Guidelines lies beyond alopecia. We hope to create a philosophy and a symbol of our resolution to provide only the most authentic information to patients - the resolution that should be shared by all the fields in healthcare: We never allow manipulation.

We do believe our colleague caregivers. Only a handful of them have been seduced by Lucifer and made us all look culpable. It is time to stop them and regain the trust of our patients, our most precious beings. At the end of the day, we will say that "once upon a time, treatment result manipulation did occur, but that is all history now." You know what we mean, and we know you are with us.

\section{Acknowledgement}

None.

\section{Conflict of interest}

None.

\section{References}

1. Wade N. It may look authentic; here's how to tell it isn't. The New York Times. 2006. 24 p.

2. Rossner M, Yamada KM. What's in a picture? The temptation of image manipulation. J Cell Biol. 2004;166(1):11-15.

3. Blatt M, Martin C. Manipulation and misconduct in the handling of image data. Plant Cell. 2013;25(9):3147-3148.

4. Wilde O, Beckson KE. I Can Resist Everything Except Temptation: And Other Quotations from Oscar Wilde. New York: Columbia University Press; 1997. 198 p.

5. Unger W, Cole J. Hair transplantation. New York. 1995.

6. Illuminating Engineering Society of North America, Lighting for Hospitals and Health Care Facilities (ANSI Approved American National Standard: ANSI/IESNA RP 29-06). New York: Illuminating Engineering Society of North America; 2006.

7. Zarei M, Falto-Aizpurua L. Low level laser therapy and hair regrowth: an evidence-based review. Lasers in Medical Science. 2016;31(2):363371.

8. Kaufman KD, Elise A Olsen, Whiting D, et al. Finasteride in the treatment of men with androgenetic alopecia. JAAD. 1998; 39(4):578589.

9. Olsen EA, Dunlap FE, Funicella T, et al. A randomized clinical trial of $5 \%$ topical minoxidil versus $2 \%$ topical minoxidil and placebo in the treatment of androgenetic alopecia in men. J Am Acad Dermatol. 2002;47(3):377-385.

10. Tomasone JR, Chaudhary R, Brouwers MC. Effectiveness of guideline dissemination and implementation strategies on health care professionals' behaviour and patient outcomes in the cancer care context: a systematic review protocol. Systematic Reviews. 2015;4(1):1-5.

11. Prior M, Guerin M, Grimmer-Somers K. The effectiveness of clinical guideline implementation strategies: a synthesis of systematic review findings. J Eval Clin Pract. 2008;14(5):888-897. 
12. Gagliardi AR. "More bang for the buck": exploring optimal approaches for guideline implementation through interviews with international developers. BMC Health Services Researcch. 201212(1):404.

13. Gagliardi AR, Brouwers MC, Bhattacharyya OK. The guideline implementability research and application network (GIRAnet): an international collaborative to support knowledge exchange: study protocol. Implementation Science. 2012;7:26.
14. Jeong HJ, Jo HS, Oh MK, et al. Applying the RE-AIM Framework to Evaluate the Dissemination and Implementation of Clinical Practice Guidelines for Sexually Transmitted Infections. J Korean Med Sci. 2015;30(7):847-852.

15. Pronovost P, Vohr E. Safe patients, smart hospitals: how one doctor's checklist can help us change health care from the inside out. New York: Penguin; 2010. 